\title{
Application of Virtual Reality Technology to Sports
}

\author{
Ting Liao \\ Sports training Dept., Wuhan Sports University, Wuhan 430079, China
}

\begin{abstract}
As an emerging product of highly developed modern computer technology, virtual reality technology has drawn great attention from the society since its date of birth. Within just a few decades, tremendous progress has been made. It has gradually turned into a new field of science, and been extensively utilized in various fields including military, entertainment, gaming, medical, remote control robot, and education. Besides, the great application value in the field of sports forms its unique characteristics, e.g. interaction, immersion and multi-sensory. In order to indicate the direction for the follow-up study, we have reviewed the application of virtual reality technology in sports field.
\end{abstract}

Keywords-virtual reality technology; application studies; sports .

\section{INTRODUCTION}

Combined with relevant science and technology, virtual reality technology simulates a real environment with visual, hearing, touching and other features, with computer technology as the core. Users are able to interact with each other by necessary equipment and objects in digital environment, experiencing corresponding feelings and experiences similar to a real environment. Virtual reality technology, as an emerging technology, has exerted a profound impact on education. Previous ideas and models of teaching are changed owing to virtual reality as a rejection and deconstruction of reality in some traditional teaching models. Virtual reality technology presents infinite possibilities in the development of educational technology to overcome the barrier in traditional teaching and lead to updating current teaching methods.

As an emerging product of highly developed modern computer technology, virtual reality technology has drawn great attention from the society since its date of birth. Within just a few decades, tremendous progress has been made. It has gradually turned into a new field of science, and been extensively utilized in various fields including military, entertainment, gaming, medical, remote control robot, and education. Besides, the great application value in the field of sports forms its unique characteristics, e.g. interaction, immersion and multi-sensory. At present, virtual reality research in the field of sports mainly covers the following 4 aspects:

\section{APPLICATION OF VIRTUAL REALITY TECHNOLOGY}

A. Virtual Reality Technology in Competitive Sports Coaches' training purpose and athletes' training process are demonstrated to participants (coaches, organizers and athletes) during competitive sports training, in forms of multiple perceptive modes through virtual reality technology. Thus, participants can naturally interact with the simulation system to enhance their scientific training levels. Researchers in Amusitronix developed VR Baseball, VR Golf, VR Kayaking, VR Tennis, VR Snow $\backslash$ Skate $\backslash$ Surf, and other systems ${ }^{[1]}$. In the modified version of Alive system, Becker and other researchers established a Tai Chi training machine with profound information. This system adopts an implicit model of Markov to identify user's posture with a recognition rate of over $90 \%$ for 18 postures. When the recognition score of a gesture is low, the system will consider this part of motion segment different from the coaches'. An immersion sports training system based on VR technology is also achieved by Yang et al., with the core idea of intuition-based interactive method-Just Follow Me (JFM)-being compared to ghost. During training, trainers' movements are visualized in real time (initially the visual images of trainers and trainees are coincident) by the ghost metaphor. Trainees keep in steps with the ghost-trainer as close and fast as possible ${ }^{[3]}$. A sled race device was developed by American Image Company and University of California Davis, which can bring athletes into a simulated world. Three-dimensional image can turn a dark training room into a race field. Athletes on this device can truly experience the feelings of fast driving a sled along the stretch. Detecting the physical condition of athletes at any time, laboratory equipment can perform technical analysis and functional diagnosis, predict competition problems that may arise, develop the best strategy and design the best route to improve athletic performance.

In order to effectively apply the virtual reality technology to the diving games, Wang Zhaoqi, a researcher in the Institute of Computer Sciences, created a video-based tracking method of non-contact human motion to reasonably and accurately capture the motion of athletes as one of the key technical challenges ever. In addition, he also took the lead in contrasting the simulated action of athletes. With actual implementation actions on the same or split screens with the aid of video processing software, a three-dimensional human motion simulation and video analysis for sport training were achieved. According to the characteristics of trampoline movement game, Sun Yongchao installed 12 dedicated cameras in different directions of Chinese trampoline team training hall. Using a method of collecting active tracking data, he created a huge database of Trampoline technology, which laid a foundation 
for the utilization of virtual reality technology in competitive trampoline games. Professor Zhang Lin tried to develop a three-dimensional simulation of basketball. Meanwhile, with Maya software, he produced virtual stadium environment and edited composites of non-linear technical action to achieve a three-dimensional rendering and animation output, providing a technical support to optimize the skill level of basketball players . The Sport Simulation Laboratory in the Sports Science Institute of State Sports General Administration developed a training system of virtual board sailing. This system is real-time three-dimensional visual, and it constructed a realistic training virtual environment by means of a variety of field data collected by sensors, as well as a mathematical model of computing. Consequently, the training missions can even be fulfilled without a proper condition, while analysis on a certain athlete can also be evaluated. Through literature analysis, it is found that the current virtual reality applications on athletic events largely concentrates on some complicated physical games, such as trampoline, diving, and gymnastics, as well as a few skilled adversarial games such as football, basketball, boxing and other related research.

\section{B. Virtual Reality Technology in Sports Rehabilitation}

In recent years, with the application of virtual reality technology, satisfying effect has been achieved in various aspects of sports rehabilitation such as attention deficits, spatial perception disorders, memory disorders in cognitive rehabilitation, anxiety, depression, phobia in emotional disorders, akinesia, poor coordination and balance, chorea in mental diseases ${ }^{[4]}$. VR was first used in the virtual reality systems for balance training, including a stationary bike and a flat panel display supplying visual virtual environment. It is found that after a period of cycling training in the virtual space, the patient's postural balance control had been largely improved ${ }^{5]}$. A thorough study found that patients can use Gait Master 2 (GM2) virtual reality devices for gait training. Standing on discrete pedals and alternating pedals, their movement trajectory was in accordance with normal walking pace, passing normal walking proprioception to their feet. The screen provides a visual space of various virtual terrain environments. Moreover, Burdea et al. developed a rehabilitation training program controlled by ankle movement. In the virtual missions, the patient will stand on the foot pedal connected to the device, and use their feet to drive a virtual training aircraft with motion control capability. It has been proved that the utilization of virtual reality technology can speed up recovering the kinetic ability of their injured ankle. The application of virtual reality technology in China has reached the practical stage of rehabilitation. For example, an independent research in Hebei University introduced a treatment plan with virtual reality technology for exoskeleton arms and its exoskeletons rehabilitation program ${ }^{[6]}$; Tsinghua University developed a virtual straight road cycling mode that can train and restore the kinetic ability of damaged lower limbs ${ }^{[7]}$. Numerous studies show that virtual reality technology in rehabilitation training has great advantages over normal rehabilitation in the real world, which can effectively solve the limitations of traditional rehabilitation methods and improve sports rehabilitation technology.

\section{Virtual Reality Technology in Popular Sports}

Ji Qingge et al. made a large-scaled group calisthenics drill simulation system with the Sport Simulation Laboratory in the Sports Science Institute of the State Sports General Administration. This system consists of formation or graphic design system, motion generation system, and group formation or design change simulation system. It reduced the arrangement and drills cost of large-scaled group calisthenics and improved the efficiency as well. A styling product called Easy Bowling Recreation Fitness is also developed to simulate the real game pattern, where the player can have a real bowling experience. The system records movements in bowling by a camera, and the real-time physical simulation and portray technology are applied to display the game results. This kind of design has the benefits of reduced space, low cost, simple setup and flexibility. Li Xiangchen and other researchers made a virtual network Marathon: an improved and distributed online platform for fitness game designs with multiple users and athletes. With the virtual reality and sports simulation technology in Marathon system, users can have more immersive sensory enjoyment and the fun from sports, athletics, and entertainment at the same time.

\section{Virtual Reality Technology in Physical Education}

It is a completely new project to apply virtual reality technology in physical education. The traditional teaching method is still adopted by most schools in current physical education. Some schools have started multimedia teaching, while few of them have introduced virtual reality technology. This is partly because of the relatively high price of hardware devices. Virtual reality technology is not yet widespread, but many advocates of physical education innovation have realized the teaching advantages and huge potential of virtual reality technology as a new teaching medium. Some relevant theory researches have also been conducted. Yu Qianchun considers that virtual reality applied in physical education can be regarded as a leap in the technological development of physical education. It can achieve immersive scenes and build an independent learning environment. The traditional learning method of promoting learning by teaching is replaced by obtaining knowledge and skills by learners' interactions with information and environment. Virtual reality physical education can not only save teaching time, but also produce direct and actual results. Students are also trained to improve their self-training awareness and innovation ability, especially in avoiding injury accidents during physical classes, compensating for bad conditions, building virtual figures, overcoming the space and time limit, and helping unleash school potential. Sun Yuezhou and other researchers pointed out that the application of virtual reality technology in physical education will remarkably turn sport technique into entertainment and gain popularity, so that the physical teaching method will be more comprehensive. 


\section{DEFICIENCIES OF THE CURRENT RESEARCH}

After a comprehensive analysis of the above results, it can be found that both experts and scholars agree on the irreplaceable innovation value of virtual reality technology in sports. The achievement of this technology will bring another technological revolution in sports. Especially in the field of sports technique analysis, virtual reality technology indicates the transformation of human movement measurement from traditional eye-based observation to the capture and analysis of high-precision motion. It also indicates the transformation from empirical methods involving too much emotion to human motion simulation based on programming and human motion simulation analysis. In fact, the theoretical recognition needs to be proved and further developed by practical exploration. Currently, the research on virtual reality technology in China's sports has the following deficiencies:

\section{A. Lack of Applicative Research Combining Theory with Practice.}

Virtual reality technology can create visual movement of the whole process, and learners are provided with vivid and realistic learning environment. It can also help overcome the key and difficult points of technology and strategy and correct error, so that the technical move can be more vivid and stereo which is helpful for skill training. Few virtual reality technologies are applied in Chinese sports. There is a quantity of research about the solutions to technical difficulties, but rarely combined with physical practice. Studies of the development process are common, but researches about practice and research findings are rare.

\section{B. Lack of Practical Research on Specific Sports.}

To realize the technique and strategic assistant application of virtual reality technology, researches have to combine the reality of sport events with the laws of human science and human kinematics. Only by accurately positioning in the early stage, understanding the users, and clarifying relationship, can its practicality be achieved successfully. None of the existing fulfillments such as trampoline, diving, gymnastics, basketball, etc., can achieve this requirement. Their researches are generalized through collaboration with related sports experts, which do not meet the requirements of athletic trainings or competitions, and fail to support the sports teaching.

\section{Lack of Development and Applicative Research in the Field of Physical Education.}

Education is the foundation for the development of all areas. The emphasis on physical education and cultivating innovative and compound talents in sports can ensure the progressiveness of sportsmanship spirits, promotion of mass sports, and the talents reservation for competitive sports. However, in current field of sports education, the development and utilization of modern approaches are much limited due to the lack of investment funds, space, time and other constraints. It is an obligation for physical education researchers to figure out the answers to the following three questions: How can modern technology be integrated and accepted with the available resources? How can interdisciplinary collaboration and innovation be strengthened to continuously improve teaching methods and optimize teaching content? How can the teaching efficiency be improved to maximize students' benefits?

\section{REFERENCES}

[1] Amusitronix. VR Sports [OL]. http://www.amusitronix.com/VR sport.html, 2003

[2] Becker D, Pentland A. Using a virtual environment to teach caner patients to Tai Chi[A]. In: Proceedings of International Conference on Automatic Face and Gesture Recognition, Killington, Vermont, 1996.157 162.

[3] Yang U Y, Kim G J. Implementation and evaluation of "Just Follow Me": An immersive, VR-based, motion training system [J]. Presence: Tele operators and Virtual Environments, 2002,11(3):304 323

[4] Rizzo AA, Schultheis M, Kerns KA, et al. Analysis of assets for virtual reality applications in neuropsychology [J].Neuropsychology Rehabil 2004:14(1/2):207 309.

[5] Kizony R, Raz L, Katz N, ct al. Video-capture virtual reality system for patients with parapl-egic spinal cord injury. [J]JRRD,2005.42(5):595 608.

[6] Yano H, KasaiK, Saitou H, et al. Development of a gait rehabilitation system using a locomotion interface. [J]. Visual Compute Animate. 2003,14:243 252.

[7] Burdea J E, Latonio J, Burdea GC, et al. Post-stroke rehabilitation with the Rutgers Ankle system: a case study. Presence 2001,10:416 430. 